\title{
Developing InP-based solar cells: Time-resolved terahertz measurements of photoconductivity and carrier multiplication efficiencies
}

DOI:

10.1109/ICIMW.2010.5612377

Link to publication record in Manchester Research Explorer

Citation for published version (APA):

Spencer, B. F., Graham, D. M., Lange, C., Chattopadhyay, S., \& Flavell, W. R. (2010). Developing InP-based solar cells: Time-resolved terahertz measurements of photoconductivity and carrier multiplication efficiencies. In IRMMW-THz 2010 - 35th International Conference on Infrared, Millimeter, and Terahertz Waves, Conference Guide/IRMMW-THz - Int. Conf. Infrared, Millimeter, Terahertz Waves, Conf. Guide IEEE. https://doi.org/10.1109/ICIMW.2010.5612377

Published in:

IRMMW-THz 2010 - 35th International Conference on Infrared, Millimeter, and Terahertz Waves, Conference Guide|IRMMW-THz - Int. Conf. Infrared, Millimeter, Terahertz Waves, Conf. Guide

\section{Citing this paper}

Please note that where the full-text provided on Manchester Research Explorer is the Author Accepted Manuscript or Proof version this may differ from the final Published version. If citing, it is advised that you check and use the publisher's definitive version.

\section{General rights}

Copyright and moral rights for the publications made accessible in the Research Explorer are retained by the authors and/or other copyright owners and it is a condition of accessing publications that users recognise and abide by the legal requirements associated with these rights.

Takedown policy

If you believe that this document breaches copyright please refer to the University of Manchester's Takedown Procedures [http://man.ac.uk/04Y6Bo] or contact uml.scholarlycommunications@manchester.ac.uk providing relevant details, so we can investigate your claim.

\section{OPEN ACCESS}




\title{
Developing InP-based solar cells: time-resolved terahertz measurements of photoconductivity and carrier multiplication efficiencies
}

\author{
B. F. Spencer ${ }^{1,2}$, D. M. Graham ${ }^{1}$, C. Lange ${ }^{3}$, S. Chattopadhyay ${ }^{1,2}$, and W. R. Flavell ${ }^{1}$ \\ ${ }^{1}$ School of Physics and Astronomy and the Photon Science Institute, The University of Manchester, \\ Manchester M13 9PL, United Kingdom \\ ${ }^{2}$ Cockcroft Institute, Daresbury Science and Innovation Campus, Warrington, Cheshire WA4 4AD, \\ United Kingdom \\ ${ }^{3}$ Department of Physics, University of Toronto, 60 St. George Street, Toronto ON, M5S 1A7, Canada
}

\begin{abstract}
Time-resolved terahertz spectroscopic studies were carried out on bulk InP to measure photoconductivity on the picosecond timescale. Comparison of the carrier multiplication quantum yields obtained with those of colloidal InP nanoparticles clearly demonstrate the potential benefit of InP nanoparticle-based next-generation solar cells.
\end{abstract}

\section{INTRODUCTION}

$\mathrm{W}$ $\mathrm{E}$ are currently in a global energy crisis, with the global demand for energy set to double by 2050. It is crucial to develop more efficient and cost effective renewable energy sources: the amount of solar energy incident upon the Earth in one hour is equivalent to the global energy consumption in one year [1], and so the utilization of solar power is a rapidly growing research area.

The next-generation of solar energy cells could be fabricated from semiconductor nanoparticles such as colloidal quantum dots. Not only do these quantum dots offer cheaper fabrication, but they may also provide a means of exceeding the Shockley-Queisser efficiency limit [2]. Where photon energy in excess of the band-gap in traditional silicon solar cells is lost as heat, providing a theoretical limit to the efficiency of $\sim 31 \%$, quantum dots may, due to their strong quantum confinement of carriers, convert this excess energy into additional carriers, allowing potential solar cell efficiencies of up to $44 \%$ [3], a phenomenon known as 'carrier multiplication'.

Indium phosphide (InP) is of particular interest as it is a relatively benign material in comparison with other colloidal quantum dots that typically include arsenic, cadmium or lead, and is a promising alternative to silicon in the fabrication of bulk solar cells based on pn junctions [4]. We have recently used ultrafast transient absorption spectroscopy to show that carrier multiplication occurs in colloidal InP quantum dots [5]. It is, however, vital to obtain accurate values of the yield of photoexcited carriers in bulk materials in order to assess the influence of quantum confinement.

Measurements of photoconductivity in bulk materials typically require the use of electrodes. However, these measurements might be perturbed by the scattering and recombination of carriers as they travel large distances to be detected. Instead, time-resolved terahertz spectroscopy has recently been shown to be an ideal probe of carrier multiplication quantum yields, due to the picosecond time resolutions ohtained and the sensitivity of terahertz radiation 978-1-4244-6656-6/10/\$26.00 C2010 IEEE to photoexcited carriers [6]. It therefore provides an ultrafast measurement of carrier multiplication quantum yields analogous to ultrafast transient absorption spectroscopy.

\section{EXPERIMENTAL}

Terahertz (THz) pulses were generated using the $800 \mathrm{~nm}$ output from a regenerative amplifier (giving $100 \mathrm{fs}$ pulses at a repetition rate of $1 \mathrm{kHz}$ ) incident on a nonlinear $1 \mathrm{~mm}$ thick (110) ZnTe crystal. Optical rectification leads to the emission of radiation at terahertz frequencies due to difference frequency mixing within the spectral bandwith of the femtosecond laser pulses. A silicon filter was used after the generation crystal to remove any residual $800 \mathrm{~nm}$ radiation. The terahertz beam was focused onto the sample using an array of gold off-axis parabolic mirrors, to give a spot size of $3 \mathrm{~mm}\left(1 / \mathrm{e}^{2}\right)$ at the sample position, measured with a knifeedge beam profiler. A schematic diagram of the experiment is illustrated in Figure 1.

The THz pulses were detected electro-optically. An 800 $\mathrm{nm}$ detection beam is brought collinear with the $\mathrm{THz}$ beam using a piece of indium tin oxide coated glass that acts as a dichroic mirror [7]. The detection beam then copropagates with the $\mathrm{THz}$ beam through a second $0.5 \mathrm{~mm}$-thick (110) $\mathrm{ZnTe}$ crystal. A phase modulation is induced in the detection beam depending on the electric field of the $\mathrm{THz}$ radiation (Pockells effect). A quarter wave-plate then converts the polarization of this detection beam from linear to circular polarization. A polarizing beam splitter allows the magnitude of the two orthogonal components of the polarization of the detection beam to be measured using a pair of balanced photodiodes. A lock-in amplifier is used to measure the difference between the polarization components: when the $\mathrm{THz}$ beam is modulated with a chopper (CH1 in Figure 1) synchronized to a sub-harmonic of the regenerative amplifier repetition rate, the lock-in amplifier detects the change in the diode signals at the chopping frequency. $\mathrm{A} \mathrm{THz}$ waveform is measured by sampling different parts of the electric field by varying the time delay between the $\mathrm{THz}$ and detection beams with an optical delay line, DL1 (Figure 1).

The experiment is fully automated with a LabView data acquisition programme. The experiment is purged with dry air to less than $5 \%$ relative humidity to minimize $\mathrm{THz}$ absorption by atmospheric water vapour.

The measurements required to extract photoconductivity 


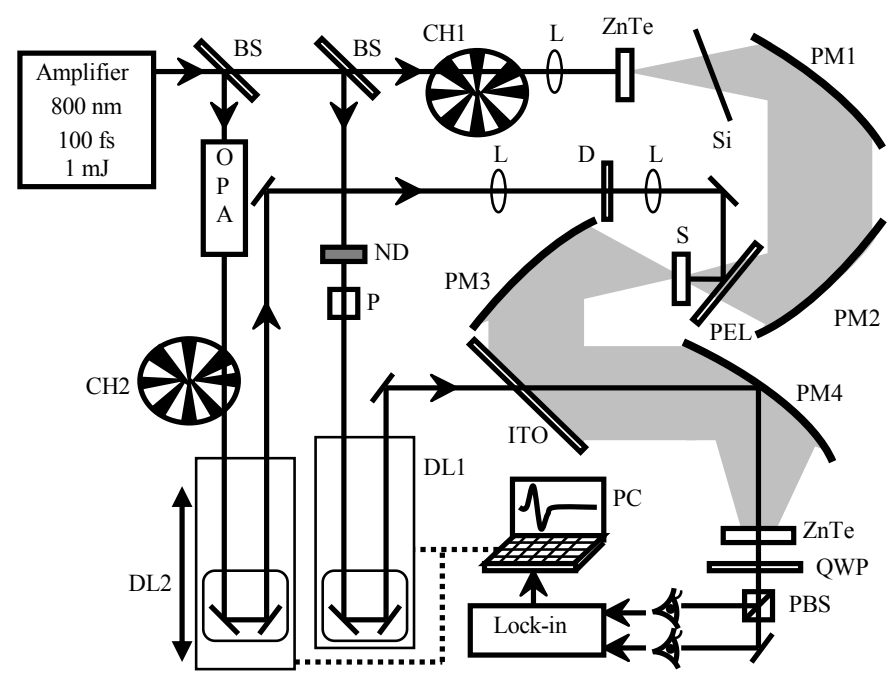

Figure 1. Time-resolved $\mathrm{THz}$ spectrometer. $\mathrm{BS}$ : beam splitter; $\mathrm{CH}$ : chopper; L: lens; ND: neutral density filter; P: polarizer; D: diffuser; Si: high-resistivity silicon filter; PM: 90 degree off-axis gold parabolic mirror; PEL: pellicle beam splitter; S: sample; DL: delay line; ITO: indium tin oxide coated glass dichroic mirror; OPA: optical parametric amplifier; QWP: quarter wave-plate; PBS: polarizing beam splitter; PC: automation computer.

are (i) the $\mathrm{THz}$ waveform without the sample, (ii) the $\mathrm{THz}$ waveform with the sample in place, and (iii) the difference in the $\mathrm{THz}$ waveform $(\Delta E(t))$ when the pump beam is used to photoexcite the sample. This $\Delta E(t)$ scan is obtained by chopping the pump beam with chopper $\mathrm{CH} 2$ and scanning delay line DL1. Pump scans (chopping the pump beam and scanning delay line DL2) were also taken to measure the evolution of the system over time, where the decay of the difference signal over time, as carriers cool and recombine, is measured as a function of the time delay between pump and probe, $\tau$.

The InP sample was a 2-inch diameter, $0.5 \mathrm{~mm}$ thick, semi-insulating wafer (Fe-doped), polished on one side. The high resistivity of the sample allowed transmission of the $\mathrm{THz}$ radiation that would otherwise be heavily attenuated by free carrier absorption. The sample was photoexcited using an optical parametric amplifier (OPA) with wavelengths ranging between 240 and $800 \mathrm{~nm}$ with a spot size of $10 \mathrm{~mm}$. A diffuser was used to create a homogeneous excitation beam free from potential hot spots. The sample was photoexcited at normal incidence using a pellicle beam splitter.

\section{RESULTS AND DISCUSSION}

Figure 2 shows the electric field of the THz pulses through the InP sample detected electro-optically, with a signal-tonoise ratio greater than 1000:1. The bandwidth of the power spectrum is found to be approximately $3 \mathrm{THz}$. The change in the $\mathrm{THz}$ waveform upon photoexcitation of the sample with $3.10 \mathrm{eV}$ photons is also shown, along with a pump scan of the time evolution of the peak value of $\Delta E(t)$. Following excitation, no change is seen over $20 \mathrm{ps,} \mathrm{meaning} \mathrm{that} \mathrm{no}$ significant recombination of photoexcited carriers occurs on 978-1-4244-6656-6/10/\$26.00 C2010 IEEE

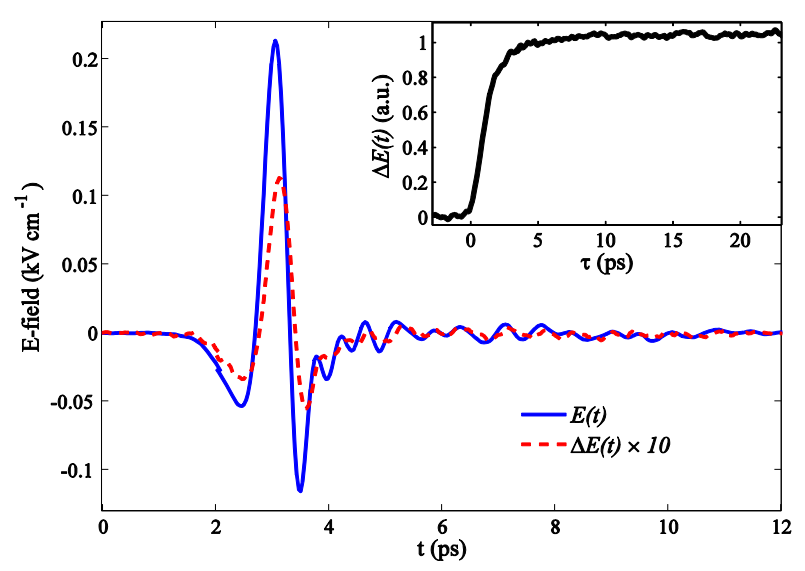

Figure 2. Electric field of the THz pulses, $E(t)$, through InP and the change in the THz pulses, $\Delta E(t)$, upon photoexcitation with $400 \mathrm{~nm}$ $(3.10 \mathrm{eV})$ radiation at a fluence of $12.5 \mathrm{~nJ} \mathrm{~mm}^{-2}$ and a pump-probe delay time, $\tau$, of $12 \mathrm{ps}$. The inset shows the evolution of this change (the maximum of $\Delta E(t))$ over time.

this timescale.

The first step in the analysis is the extraction of the complex refractive index of the sample before photoexcitation, $\tilde{n}=n+i k$. This is determined by numerically fitting the transmission coefficient of the $\mathrm{THz}$ beam through the sample:

$$
\frac{E_{S}(\omega)}{E_{R E F}(\omega)}=\frac{4 \widetilde{n}}{(\widetilde{n}+1)^{2}} \exp \left[\frac{i \omega l}{c}(\widetilde{n}-1)\right]
$$

where $E_{S}(\omega)$ and $E_{R E F}(\omega)$ are the Fourier transforms of the $\mathrm{THz}$ electric fields with and without the sample, $\omega$ is angular frequency, $l$ is the sample thickness and $c$ the speed of light. A computationally fast fixed-point iteration method is implemented to extract $\tilde{n}$ [8]. Multiple reflections of the $\mathrm{THz}$ pulses from the sample surfaces are ignored here because the time-domain data are truncated by applying a temporal window to remove them.

InP is highly absorbing in the visible and ultraviolet spectral range, such that the optical penetration depth is much less than the terahertz wavelength. This creates a thin photoexcited layer at the surface exhibiting a change in refractive index $\Delta \widetilde{n}$ and a transient photoconductivity $\Delta \sigma$ :

$$
\Delta \sigma=-2 i \varepsilon_{0} \omega \tilde{n} \Delta \tilde{n}
$$

Multiple reflections of the $\mathrm{THz}$ waveform through such a thin photoexcited layer are now indistinguishable in the timedomain, and so analysis to extract $\Delta \widetilde{n}$ upon photoexcitation must take this into account.

The carrier multiplication quantum yield, $\eta$, or the ratio of photoexcited carrier to photon densities in the sample, was extracted by the method described by Pijpers et al. [6]. This method only requires knowledge of the reflectivity of the InP surface and the optical absorption coefficient, obtained from 


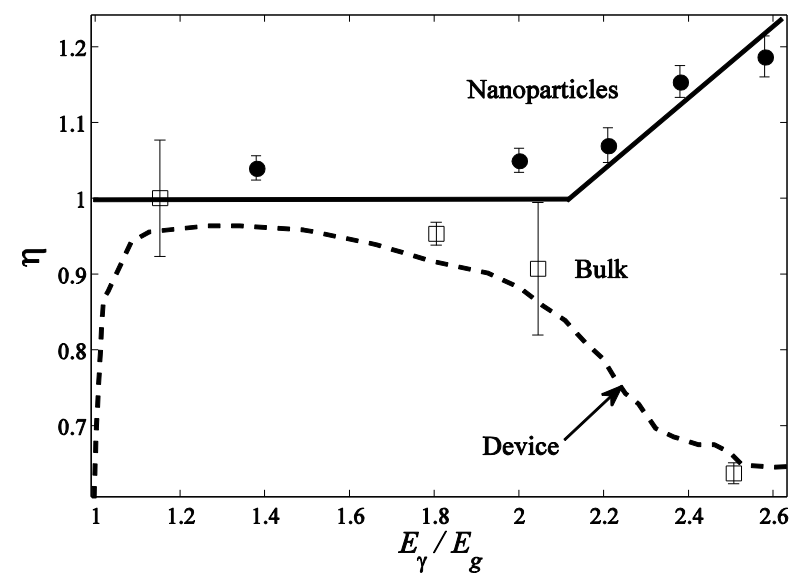

Figure 3. Carrier multiplication quantum yield, $\eta$, for bulk (squares, this work) and nanoparticulate (filled circles [5]) InP, at various photon energies, $E_{\gamma}$, corresponding to multiples of the band gap energy, $E_{g}$. The bulk measurements are scaled to the first data point (800 nm pump beam) where $\eta=1$. The nanoparticle quantum yield was measured using transient absorption spectroscopy [5]. The dashed line shows the internal quantum efficiency of a bulk InP photovoltaic device [4]. In the case of the nanoparticles, the quantum yield increases linearly beyond the carrier multiplication threshold (2.1 $E_{g}$ ), illustrated by the solid line (a guide to the eye).

Aspnes et al. [9].

Carrier multiplication, where photon energy in excess of the band gap is utilized to create additional charge carriers, has been demonstrated in a number of different nanoparticles including InAs [10], InP [5] and PbS [11]. However, it has been shown that the carrier multiplication quantum yields for $\mathrm{PbS}$ quantum dots may be less than those of the bulk material [6]. Similar measurements on other materials are required to fully understand the effect of quantum confinement on the quantum efficiency of a material.

Figure 3 shows a comparison of the carrier multiplication quantum yields, $\eta$, for bulk InP obtained through this work with those previously obtained by us for nanoparticulate InP quantum dots [5]. The latter had a band gap of $2.0 \mathrm{eV}$ (absorption edge at $620 \mathrm{~nm}$ ) in comparison to $1.34 \mathrm{eV}$ for bulk InP at $300 \mathrm{~K}$. In order to compare these materials accurately, the carrier multiplication quantum yield is plotted against photon energy in terms of a multiple of the band gap of the material rather than absolute photon energy. The results obtained for bulk $\mathrm{InP}$ show that carrier multiplication quantum yields decrease at high photon energies, in agreement with measurements from bulk InP photovoltaic devices [4]. This contrasts with an increase in quantum yield observed in InP quantum dots [5]. Importantly, we find no evidence from terahertz spectroscopy for carrier multiplication in bulk InP (i.e. $\eta \leq 1)$ The threshold for carrier multiplication in the InP quantum dot sample was measured to be $2.1 E_{g}$ [5], which is the theoretical threshold for the process as required by the conservation of energy [12].

Transient absorption spectroscopy was used to extract the carrier multiplication quantum yields of the InP quantum dots shown in Figure 3 [5]. This technique uses a white-light continumm inter-hand excitation nrohe as nnnosed to a $\mathrm{TH} 7$ 978-1-4244-6656-6/10/\$26.00 (2010 IEEE probe which would be absorbed through sub-band transitions in bound electron-hole pairs (excitons) as well as by phonon modes.

Application of terahertz spectroscopy to InP quantum dots is the next step in this research, in order to further understand the physical processes involved in carrier cooling. The main challenge in this will be to increase the signal-tonoise ratio such that much smaller changes in the $\mathrm{THz}$ signal can be detected. A greatly reduced absorption of $\mathrm{THz}$ radiation is observed in quantum dot samples because the quantum confinement of semiconductors leads to a low phonon density of states, and so fewer phonon modes may be excited. The number of excitons present in a quantum dot sample (typically held in solution with hexane) will also be dramatically less than in a bulk material [13].

Figure 3 illustrates the benefit that next-generation solar cells based on InP quantum dots could have on solar power. Solar energy cells based on quantum dots also have other benefits. Not only is the manufacturing cost of quantum dots heavily reduced in comparison to traditional silicon solar cells, but also the band gap of quantum dots can be tuned by adjusting the size of the quantum dot. The absorption of a quantum dot utilizing the carrier multiplication process can therefore be adjusted to best overlap with the solar spectrum.

\section{CONCLUSION}

Time-resolved $\mathrm{THz}$ spectroscopy has been used to measure the carrier multiplication yield in bulk InP. These yields decrease at high photon energies in bulk InP, in contrast to the increase in quantum yield observed in InP quantum dots [5]. This demonstrates the benefit that InP quantum dots could have on the utilization of solar power when implemented in a next-generation solar cell.

\section{ACKNOWLEDGEMENTS}

This work was carried out with the financial support of the British Council and the German Academic Exchange Service (DAAD) through the Academic Research Collaboration (ARC) programme (Grant No. 1362).

\section{REFERENCES}

[1] O. Morton, Nature 443, 19 (2006).

[2] W. Shockley and H. J. Queisser, J. Appl. Phys. 32, 510 (1961).

[3] M. C. Hanna and A. J. Nozik, J. Appl. Phys. 100, 074510 (2006).

[4] H. J. Schimper et al., J. Crys. Growth 287, 642 (2006).

[5] S. K. Stubbs et al., Phys. Rev. B 81, 081303(R) (2010).

[6] J. J. H. Pijpers et al., Nature Physics 5, 811 (2009).

[7] T. Bauer et al., J. Appl. Phys. 92, 2210 (2002).

[8] W. Withayachumnankul et al., Proc. SPIE Photonic Mater., Dev., and App. 5840, 221 (2005).

[9] D. E. Aspnes and A. A. Studna, Phys. Rev. B 27, 985 (1983).

[10] R. D. Schaller, Nano Letters 7, 3469 (2007).

[11] R. J. Ellingson et al., Nano Letters 5, 865 (2005).

[12] M. C. Beard and R. J. Ellingson, Laser Photonics Rev. 2, 377 (2008).

[13] E. Hendry et al., Phys. Rev. Letters 96, 057408 (2006). 